\title{
INDIRECT MEASURES OF NEUROPROTECTION ARE PARAMETERS TO AVOID: EXAMPLES FROM RESEARCH ON NEONATAL RAT HYPOXIA-ISCHEMIA
}

\author{
DOROTHY E OORSCHOT \\ Department of Anatomy and Structural Biology, and the Brain Health Research Centre, University of Otago, \\ Dunedin, New Zealand. \\ e-mail: dorothy.oorschot@anatomy.otago.ac.nz \\ (Accepted March 7, 2011)
}

\begin{abstract}
The use of indirect measures of neuronal injury or neuroprotection, such as brain weight or the neuronal density of neurons, can lead to misinterpretations of biological processes. The use of modern stereological methods to directly measure the absolute number of surviving neurons generally yields more reliable conclusions. The aim of this review is to provide some worked examples of this principle. The worked examples are obtained from research completed over the past 13 years on the investigation of potential neuroprotective therapies for moderate brain injury after neonatal hypoxia-ischemia in the rat.
\end{abstract}

Keywords: absolute neuronal number, brain weight, Cavalieri's method, neuronal density, optical disector, stereology.

\section{INTRODUCTION}

The use of indirect measures of neuronal injury or neuroprotection, such as brain weight or the neuronal density of neurons, can lead to misinterpretations of biological processes (Gundersen, 1986; Swaab and Uylings, 1987; Oorschot 1994; West, 1999; Boyce et al., 2010). The use of modern stereological methods to directly measure the absolute number of surviving neurons generally yields more reliable conclusions (Gundersen, 1986; Swaab and Uylings, 1987; Oorschot 1994; West, 1999; Boyce et al., 2010). The aim of this review is to provide some worked examples of this principle. The worked examples are obtained from research completed over the past 13 years on the investigation of potential neuroprotective therapies for moderate brain injury after neonatal hypoxia-ischemia in the rat.

Exposure to low levels of oxygen (i.e., hypoxia) and decreased blow flow (i.e., ischemia) around the time of birth (i.e., neonatally) is a major cause of acute brain injury that can lead ultimately to cerebral palsy, mental retardation, epilepsy and learning deficits (Perlman, 2006). Injury to nerve cells in the striatum of the brain is likely to contribute to the motor deficits seen in cerebral palsy (Volpe, 2001a). Injury to nerve cells in the hippocampus of the brain may contribute to the cognitive impairments (Volpe, 2001b). Potential neuroprotective therapies have been investigated that target one or more of the biochemical pathways that are thought to contribute to the death of nerve cells following exposure to neonatal hypoxia-ischemia. These therapies include treatment with magnesium sulfate (Galvin and Oorschot, 1998), growth factors (Galvin and Oorschot, 2003), antioxidants (Covey et al., 2006), moderate hypothermia (Covey and Oorschot, 2007), and treatment with an antioxidant in combination with moderate hypothermia (Hobbs and Oorschot, 2008; Oorschot et al., unpublished observations). In an animal model of neonatal hypoxia-ischemia that has been used in these studies, the right common artery has been permanently ligated in the neck. Each neonatal rat is then exposed to hypoxia for $1.5 \mathrm{hrs}$ to yield an injury that is primarily on the right side of the brain.

Various methods have been used to measure neuronal survival in studies investigating potential neuroprotective therapies. Indirect measures that have been used include measurement of the weight of the entire brain, the weight of a specific subregion of the brain (e.g. the right or left cerebral hemisphere), and the total or absolute volume of a specific part of the cerebral hemisphere (e.g. the striatum). Other indirect measures that have been used include the volume of lesioned or injured brain tissue, qualitative 
scoring of brain injury, and quantitative measurement of the number of neurons in 1-4 sections, or in a subvolume, of the entire structure (i.e., the neuronal density). Direct measurement of the absolute number of surviving nerve cells can be determined using modern stereological methods. The absolute volume (i.e., the total reference volume or $\mathrm{V}$ (ref) or volume (V)) of the brain structure of interest (e.g. the striatum) can be multiplied by the number of neurons in a known subvolume $\left(\mathrm{N}_{\mathrm{v}}\right.$, i.e., the neuronal density) to yield the absolute number of surviving nerve cells. More specifically, the absolute volume can be measured using Cavalieri's method (Gundersen and Jensen, 1987; Gundersen et al., 1999) and the $\mathrm{N}_{\mathrm{v}}$ can be measured using the optical disector method (Gundersen, 1986). In the worked examples in this review, these specific stereological methods have been used to measure the absolute number of surviving nerve cells.

To measure the absolute volume of the brain region of interest (e.g. the striatum) using Cavalieri's method, the right cerebral hemisphere of each rat in the worked examples was preserved and embedded in Technovit. Each hemisphere was serially sectioned on a microtome, with every $4^{\text {th }}$ section collected and mounted on a slide. Every $4^{\text {th }}$ section was then stained with cresyl violet to permit identification of the striatum and of the neurons within the striatum. Specific criteria were used to identify the striatum, from its anterior pole to its posterior pole, in the sections (see Fig. 1a, and Oorschot (1996) for the specific details). The sections containing the striatum were sampled systematically (e.g. every $8^{\text {th }}$ section) after a random start (Fig. 1a). The cross-sectional area of the striatum in each sampled section was then measured by point counting. Each sampled section was projected onto a white screen at a known magnification, and a lattice of regularly arranged points
(Fig. 1a) was superimposed randomly over it. The number of points falling on the striatum were counted, and the points $(\mathrm{P})$ for each section were then summed $\left(\sum \mathrm{P}\right)$. The total reference volume, $\mathrm{V}(\mathrm{ref})$, was then determined by $\mathrm{V}(\mathrm{ref})=\sum \mathrm{P} \cdot \mathrm{a}(\mathrm{p}) \cdot t$, where $a(p)$ is the real area that each point represents and $t$ is the distance between the sampled sections (Gundersen and Jensen, 1987).

To obtain the $\mathrm{N}_{\mathrm{v}}$ using the optical disector method, the sections used were the same set of sections that were used for Cavalieri's estimate of the total reference volume of the striatum. Each sampled section was viewed using a x100 oilimmersion objective (NA 1.4) on an Olympus BH-2 microscope. The microscope was also equipped with an automated mechanical stage, an electronic microcator (to measure height in the $\mathrm{z}$ plane), and a videocamera that projected the image onto an adjacent TV monitor. Overlying the TV screen was a transparent acetate sheet with an unbiased sampling frame drawn on it (Gundersen, 1977). With the sampling frame and the automated stage, the entire striatum in each selected section was sampled using a random start and systematic sampling (e.g., every 10th area) thereafter (see Fig. 1b). For each sampled area, the number of neuronal nuclei that came into focus through the middle $15 \mu \mathrm{m}$ of the section and did not fall on the sampling frame exclusion lines were counted (see Fig. 1c). The number of neurons (Q-) in a disector volume, V(dis), of tissue was thus obtained. V(dis) was determined from the area (A) of the sampling frame (i.e., a(frame) in Fig. 1b,c, corrected for magnification, x3,400) multiplied by the disector height $(h$; of $15 \mu \mathrm{m})$. The total value for $\mathrm{N}_{\mathrm{v}}$ for the striatum of an individual animal was then determined from $\mathrm{N}_{\mathrm{v}}=\sum \mathrm{Q}-/ \sum \mathrm{V}$ (dis). The absolute number of neurons, $N$, for the striatum within each individual was then calculated from $N=\mathrm{V}(\mathrm{ref}) \mathrm{x} \mathrm{N}_{\mathrm{v}}$. 


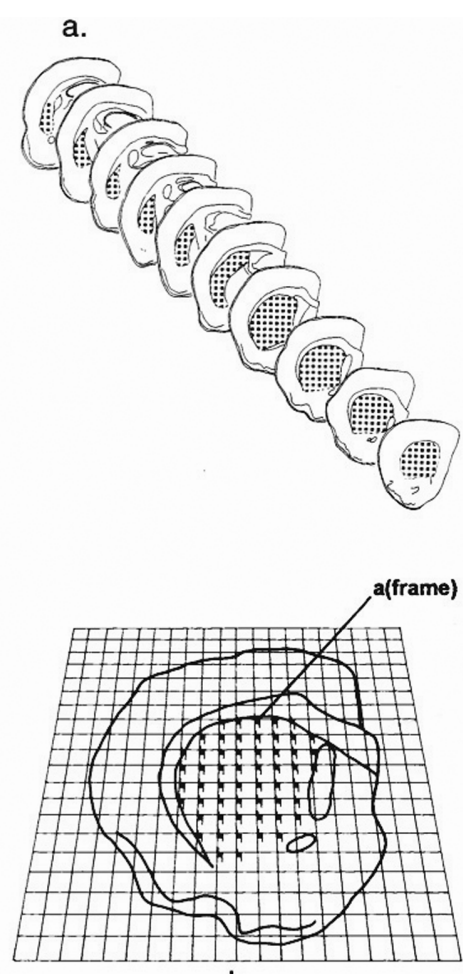

b.

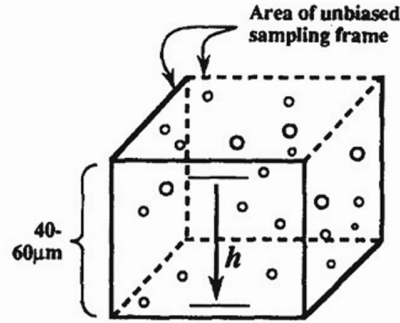

c.

Fig. 1. Schematic illustrations of a worked example through the rat striatum of Cavalieri's method $(a, b)$ and the optical disector method $(b, c)$. In (a) a systematic series of sections through the striatum, after a random start, is illustrated. A systematic array of points (i.e. dots) is overlayed on the striatum and the number of points $(P)$ falling on the striatum in each section are counted. These points are then summed to obtain the $\sum P$ for the entire striatum in a specific cerebral hemisphere. The value for $\sum P$ is substituted into Cavalieri's formula to calculate the absolute volume (see text for further details). In (b) systematic sampling throughout the striatum, after a random start, is illustrated. This strategy is used to measure the Nv in a specific section. The a(frame) refers to the area of one unbiased sampling frame, among a number of these frames, within the striatal boundaries of one section. The Nv of neurons was measured within each a(frame) and its disector height. This analysis to estimate the $N v$ was completed for all the sampled sections in (a). The counting of disector neurons through the height (h) of a sampled disector volume is illustrated in (c). (a, b) Modified from West et al. (1996), with permission. (c) From Oorschot (1994), with permission.

\section{Indirect measures, such as brain weight and qualitative scoring, may not necessarily reflect the total, or absolute, number of surviving neurons in the injured brain}

Indirect measures for determining neuronal survival, such as brain weight or qualitative scoring of injury, may not necessarily reflect the total, or absolute, number of surviving neurons in the injured brain (Oorschot, 1994). This is because brain weight is likely to reflect glial cell dynamics as well as neuronal cell dynamics post-injury. As an example of the effect of glial cell dynamics, there was only a $9 \%$ reduction in the weight of the right cerebral hemisphere after neonatal hypoxia-ischemia, compared to uninjured control animals, in a study investigating the neuroprotective potential of magnesium sulfate versus sodium chloride (Table 1, Galvin and Oorschot, 1998). This is in contrast to a $45 \%$ reduction in the absolute number of medium-spiny neurons in the right striatum of the brain after neonatal hypoxiaischemia compared to uninjured control animals (Table 1). Marked astrogliosis post-hypoxia-ischemia (Oorschot and Galvin, 1995) would contribute to the smaller reduction in hemisphere weight. Astrogliosis refers to an increased proliferation of a subtype of glial cells known as astrocytes. Thus, the way to be absolutely sure of the extent of neuronal injury is to directly measure the absolute number of neurons using modern stereological methods. 
Table 1. Data on the weight of the right cerebral hemisphere and the total number (N) of medium-spiny neurons in the right striatum of Sprague-Dawley rats at postnatal day 18. On postnatal day 7 the rats had been subjected to either neonatal cerebral hypoxia-ischemia (H/I, i.e. ligation of the right common carotid artery in the neck followed by 1.5 hrs of hypoxia) or sham-ligation of the right common carotid artery followed by no hypoxia (i.e. uninjured control rats). This was followed by immediate post-treatment with either magnesium sulfate $\left(\mathrm{MgSO}_{4}\right)$ or sodium chloride ( $\mathrm{NaCl}$ ). Data from Galvin and Oorschot (1998).

\begin{tabular}{|c|c|c|c|}
\hline Treatment group & Number of rats & $\begin{array}{l}\text { Weight of right cerebral } \\
\text { hemisphere }(\mathrm{g})\end{array}$ & $\begin{array}{c}N\left(10^{3}\right) \text { of striatal } \\
\text { medium-spiny neurons }\end{array}$ \\
\hline$\overline{\mathrm{H} / \mathrm{I} \mathrm{MgSO}_{4}}$ & 11 & & \\
\hline Mean & & $0.410^{*}$ & $1434 \dagger$ \\
\hline SD & & 0.026 & 316 \\
\hline $\mathrm{CV}$ & & 0.063 & 0.220 \\
\hline$\overline{\mathrm{H} / \mathrm{I} \mathrm{NaCl}}$ & 11 & & \\
\hline Mean & & $0.400 *$ & $1421 \dagger$ \\
\hline $\mathrm{SD}$ & & 0.045 & 443 \\
\hline $\mathrm{CV}$ & & 0.112 & 0.312 \\
\hline Uninjured Control $\mathrm{MgSO}_{4}$ & 4 & & \\
\hline Mean & & 0.450 & 2583 \\
\hline $\mathrm{SD}$ & & 0.024 & 287 \\
\hline $\mathrm{CV}$ & & 0.053 & 0.111 \\
\hline Uninjured Control $\mathrm{NaCl}$ & 4 & & \\
\hline Mean & & 0.440 & 2684 \\
\hline SD & & 0.021 & 167 \\
\hline $\mathrm{CV}$ & & 0.048 & 0.062 \\
\hline
\end{tabular}

Qualitative scoring of neuronal injury from a few selected sections through the brain structure of interest may not reflect significant volume changes that directly affect the total number of surviving neurons (Gundersen, 1986; Swaab and Uylings, 1987; Oorschot, 1994). For example, the usefulness of measuring the absolute number of surviving neurons was evident when comparisons were made with qualitative assessment of striatal injury after treatment with either diluent/normothermia or an antioxidant/hypothermia combination (Hobbs and Oorschot, 2008). In animals that were post-treated with diluent/normothermia immediately after neonatal hypoxic-ischemic brain injury at 1-week-of-age, there was qualitative evidence of neuronal loss in the right striatum in two of eight animals at 2-weeks-ofage and two of 12 animals at 12-weeks-of age. There was a quantitative decrease in the absolute number of medium-spiny neurons in the right striatum of six of these eight animals at 2-weeks-of-age, and in all 12 animals at 12-weeks-of age. Qualitative evidence of gliosis in the right striatum was seen in three of eight animals at 2-weeks-of-age and three of 12 animals at 12-weeks-of age. A qualitative decrease in medium-spiny neurons or gliosis in the right striatum was not evident in antioxidant/hypothermiatreated animals at 2-weeks-of age nor 12 weeks-of- age. There was a quantitative decrease in the absolute number of medium-spiny neurons in the right striatum in one of eight, and four of 12, antioxidant/ hypothermia-treated animals at 2-weeks-of age, and at 12 weeks-of-age, respectively. Quantitation was needed, therefore, to detect moderate striatal injury.

\section{Indirect measures, such as brain weight, regional volume, lesion volume, and neuronal density, may not necessarily reflect the total, or absolute, number of surviving neurons in studies on neuroprotection}

In a study investigating the neuroprotective potential of two growth factors, brain derived neurotrophic factor (BDNF) and neurotrophin-3 (NT-3), the absolute neuronal number data revealed that BDNF or NT-3 increased the total number of surviving medium-spiny neurons in the right striatum by $43 \%$ and $33 \%$, respectively (Table 2 ; Galvin and Oorschot, 2003). This significant neuroprotection was not evident, however, when brain weight, striatal volume, striatal lesion volume, and neuronal density measures for NT-3, were compared (see Table 2). The data for the striatal lesion or infarct volume were: Infarct volume for H/I BDNF- 
treated striatum, $0.09 \pm 0.10 \mathrm{~mm}^{3}$ (mean $\pm \mathrm{SEM}, \mathrm{n}=$ 8); $\mathrm{H} / \mathrm{I}$ no BDNF diluent control striatum, $0.36 \pm 0.28$ $\mathrm{mm}^{3} ; \mathrm{H} / \mathrm{I} \mathrm{NT}-3$-treated striatum, $0.23 \pm 0.16 \mathrm{~mm}^{3}$; H/I no NT-3 diluent control striatum, none of the eight animals had infarcts; (Galvin and Oorschot, 2003). When the average infarct volume was compared for each neurotrophin and its control (8 pups/group), no significant differences were apparent ( $\mathrm{p}=0.96$, respectively, Mann-Whitney $U$ test, Galvin and Oorschot, 2003). These measures therefore missed the protective effect demonstrated by the total neuronal count. This discrepancy in the biological conclusions derived from the absolute number data versus the other measures indicates that the way to be absolutely sure of the presence or absence of neuroprotection after low dose treatment and a moderate hypoxic-ischemic brain injury, at least in the short-term (i.e., after 7 days), is to measure the absolute or total number of surviving neurons using stereological methods.

It could be argued that the significantly higher neuronal density for the BDNF-treated rats versus their controls (Table 2) is indicative of neuroprotection without determining the total neuronal number. Yet, neuronal density measures in the absence of total neuronal number data can be misleading (e.g. Gundersen, 1986; Oorschot 1994; West, 1999; Boyce et al., 2010). This is evident in the current study for NT-3, where the neuronal density data indicates no neuroprotection while the total number data indicates protection (Table 2). It could also be argued that the total striatal volume data for NT-3 is close to statistical significance (Table 2) and may have achieved significance if more animals had been studied or a longer survival time had been used. Yet, the total neuronal number data revealed significant protective effects for NT-3 without needing to study more animals nor a longer survival time. Measurement of total neuronal number does take longer than measurement of total volume, yet this would only take an extra sixteen normal working days in our laboratory for a combined total of 16 rats in a treatment versus control group as in this study. This is less than the time needed to study either more animals or a longer survival period. Thus, measurement of total neuronal number was efficient in terms of detection of neuroprotection, animal ethics and time.

In another study investigating the neuroprotective potential of antioxidant/hypothermia combinatorial treatment on striatal medium-spiny neurons (Hobbs and Oorschot, 2008), if the brain weight or striatal volume been used as the sole measure of neuroprotection, the efficacy of the antioxidant, in combination with moderate hypothermia, would have been undetected in the short-term experiment (i.e., at 2 weeksof-age [PN 14], Table 2). In the long-term experiment, if the brain weight or $\mathrm{Nv}$ been used as the sole measure of neuroprotection, the efficacy of the antioxidant, in combination with moderate hypothermia, would have been undetected (i.e., at 12 weeks-of age [PN 83], Table 2).

When the neuroprotective potential of this combinatorial treatment was investigated for CA1 neurons in the hippocampus (Oorschot et al., unpublished observations), if the weight of the right cerebral hemisphere had been used as the sole measure of neuroprotection, the efficacy of the antioxidant, in combination with moderate hypothermia, would have been statistically undetected (Table 3 ). The effectiveness of combined antioxidant-hypothermia treatment was evident when the absolute number of surviving hippocampal neurons, or the $\mathrm{Nv}$ of CA1 neurons, or the total reference volume of the CA1 region, was compared. 
Table 2. Data on the weight of the right cerebral hemisphere, the volume (V) of the right striatum, and the Nv and total number $(N)$ of medium-spiny neurons in the right striatum of Sprague-Dawley rats at postnatal day (PN) 14 or PN 83. On PN 7 treatment was commenced with either BDNF or its diluent, NT-3 or its diluent, or an antioxidant or its diluent, respectively. On PN 8 the rats had been subjected to neonatal cerebral hypoxiaischemia (H/I, i.e., ligation of the right common carotid artery in the neck followed by 1.5 hrs of hypoxia). This was followed by post-treatment with either BDNF or its diluent, NT-3 or its diluent, or antioxidant/ hypothermia or diluent/normothermia, respectively. The BDNF and NT-3 data are from Galvin and Oorschot (2003) and the antioxidant/hypothermia data are from Hobbs and Oorschot (2008).

\begin{tabular}{|c|c|c|c|c|c|}
\hline Treatment group & $\begin{array}{l}\text { Number } \\
\text { of rats }\end{array}$ & $\begin{array}{l}\text { Weight of right } \\
\text { cerebral } \\
\text { hemisphere }(\mathrm{g})\end{array}$ & $\begin{array}{l}\mathrm{V}\left(\mathrm{mm}^{3}\right) \text { of } \\
\text { right striatum }\end{array}$ & $\begin{array}{c}\mathrm{Nv}\left(10^{4} / \mathrm{mm}^{3}\right) \\
\text { of medium- } \\
\text { spiny neurons }\end{array}$ & $\begin{array}{c}N\left(10^{3}\right) \text { of striatal } \\
\text { medium-spiny } \\
\text { neurons }\end{array}$ \\
\hline H-I BDNF, PN 14 & 8 & & & & \\
\hline Mean & & 0.370 & 8.980 & $22.990 *$ & $2024^{\dagger}$ \\
\hline SD & & 0.033 & 1.690 & 4.530 & 304 \\
\hline $\mathrm{CV}$ & & 0.089 & 0.188 & 0.197 & 0.150 \\
\hline H/I No BDNF Diluent, PN 14 & 8 & & & & \\
\hline Mean & & 0.390 & 8.180 & 17.520 & 1416 \\
\hline SD & & 0.040 & 2.190 & 4.660 & 430 \\
\hline $\mathrm{CV}$ & & 0.103 & 0.268 & 0.266 & 0.304 \\
\hline $\mathrm{H} / \mathrm{I}$ NT-3, PN 14 & 8 & & & & \\
\hline Mean & & 0.410 & 8.890 & 20.570 & $1846^{\S}$ \\
\hline SD & & 0.038 & 1.830 & 2.040 & 301 \\
\hline $\mathrm{CV}$ & & 0.093 & 0.206 & 0.099 & 0.163 \\
\hline $\begin{array}{l}\text { H/I No NT-3 Diluent Control, } \\
\text { PN } 14\end{array}$ & 8 & & & & \\
\hline Mean & & 0.410 & 6.890 & 20.350 & 1391 \\
\hline SD & & 0.031 & 2.080 & 1.650 & 400 \\
\hline $\mathrm{CV}$ & & 0.076 & 0.302 & 0.081 & 0.288 \\
\hline $\begin{array}{l}6 \text { day survival, } \mathrm{PN} 14, \\
\text { H/I Diluent/Normothermia }\end{array}$ & 8 & & & & \\
\hline Mean & & 0.400 & 10.030 & 21.290 & 2164 \\
\hline SD & & 0.083 & 2.370 & 2.060 & 640 \\
\hline $\mathrm{CV}$ & & 0.208 & 0.236 & 0.097 & 0.296 \\
\hline $\begin{array}{l}6 \text { day survival, } \mathrm{PN} 14, \\
\text { H/I Antioxidant/Hypothermia }\end{array}$ & 8 & & & & \\
\hline Mean & & 0.430 & 11.450 & $24.640^{+}$ & $2812^{\#}$ \\
\hline SD & & 0.048 & 1.050 & 1.620 & 219 \\
\hline $\mathrm{CV}$ & & 0.112 & 0.092 & 0.066 & 0.078 \\
\hline $\begin{array}{l}11 \text { week survival, PN } 83 \text {, } \\
\text { H/I Diluent/Normothermia }\end{array}$ & 12 & & & & \\
\hline Mean & & 0.710 & 19.540 & 9.790 & 1893 \\
\hline SD & & 0.110 & 6.660 & 1.060 & 635 \\
\hline $\mathrm{CV}$ & & 0.155 & 0.341 & 0.108 & 0.335 \\
\hline $\begin{array}{l}11 \text { week survival, PN } 83, \\
\text { H/I Antioxidant/Hypothermia }\end{array}$ & 12 & & & & \\
\hline Mean & & 0.770 & $24.000^{\pi}$ & 10.730 & $2578^{¥}$ \\
\hline SD & & 0.050 & 4.010 & 1.210 & 514 \\
\hline $\mathrm{CV}$ & & 0.065 & 0.167 & 0.113 & 0.199 \\
\hline \multicolumn{6}{|c|}{ 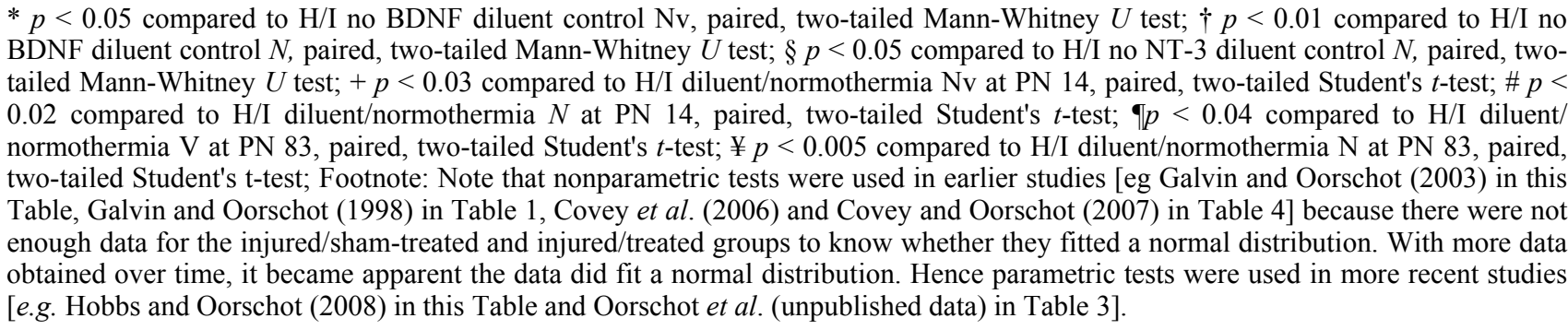 } \\
\hline
\end{tabular}


Table 3. Data on the weight of the right cerebral hemisphere, the volume (V) of the right hippocampal CA1 region, and the $N v$ and total number $(N)$ of pyramidal neurons in the right CAl region of Sprague-Dawley rats at postnatal day $(P N)$ 14. On PN 7 treatment was commenced with either an antioxidant or its diluent. On $P N 8$ the rats had been subjected to neonatal cerebral hypoxia-ischemia (H/I, i.e. ligation of the right common carotid artery in the neck followed by 1.5 hrs of hypoxia). This was followed by immediate post-treatment with either an antioxidant/hypothermia combination or diluent/normothermia. The data are from unpublished observations by Oorschot DE, Savanthrapadian S and Hobbs CE.

\begin{tabular}{lccccc}
\hline Treatment group & $\begin{array}{c}\text { Number } \\
\text { of rats }\end{array}$ & $\begin{array}{c}\text { Weight of } \\
\text { right cerebral } \\
\text { hemisphere }(\mathrm{g})\end{array}$ & $\begin{array}{c}\mathrm{V}\left(\mathrm{mm}^{3}\right) \text { of right } \\
\mathrm{CA} 1 \text { region }\end{array}$ & $\begin{array}{c}\mathrm{Nv}\left(10^{4} / \mathrm{mm}^{3}\right) \text { of } \\
\text { pyramidal neurons } \\
\text { in the CA1 region }\end{array}$ & $\begin{array}{c}N\left(10^{4}\right) \text { of pyramidal } \\
\text { neurons in the } \\
\text { CA1 region }\end{array}$ \\
\hline 6 day survival, PN 14 & 12 & & & & \\
H/I Diluent/Normothermia & & 0.425 & 0.486 & 42.997 & 21.154 \\
Mean & & 0.081 & 0.119 & 5.805 & 6.578 \\
SD & & 0.191 & 0.245 & 0.023 & 0.311 \\
CV & 12 & & & & \\
6 day survival, & & 0.453 & $0.589^{*}$ & $48.898^{\dagger}$ & $28.706^{\#}$ \\
H/I Antioxidant/Hypothermia & & 0.052 & 0.075 & 5.222 & 4.308 \\
Mean & & 0.115 & 0.127 & 0.107 & 0.150 \\
SD & &
\end{tabular}

$* p<0.03$ compared to H/I-exposed, diluent/normothermia treated control V, paired, two-tailed Student's $t$-test

$\dagger p<0.05$ compared to H/I-exposed, diluent/normothermia treated control Nv, paired, two-tailed Student's $t$-test

$\# p<0.01$ compared to H/I-exposed, diluent/normothermia treated control $N$, paired, two-tailed Student's $t$-test

When novel compounds are being investigated for their neuroprotective potential, a dose response study is recommended to determine the most effective dose and if high doses are deleterious. In a study investigating the neuroprotective potential of a novel antioxidant, MitoVitamin E (Covey et al., 2006), the significant neurotoxic effect of either pre-treatment or post-treatment with $435 \mu \mathrm{M}$ (i.e., a high dose of) MitoVitamin E was only evident when the absolute number and neuronal density of medium-spiny neurons in the right hypoxic-ischemic striatum was compared with the respective hypoxic-ischemic-diluent-treated right striatum (Table 4). It was not evident when the average weight of the right cerebral hemisphere and the total volume of the right striatum were compared between groups (Table 4). These latter indirect measures therefore missed the neurotoxic effect demonstrated by the direct measurement of neurons.

Investigation of the neuroprotective potential of therapies usually also requires an assessment of the effect on the development of the normal brain. This is needed to determine if there are any unwanted side-effects. In a study investigating the effect of moderate hypothermia on normal brain development (Covey and Oorschot, 2007), a lack of effect on normal striatal development was only evident when the absolute number of medium-spiny neurons in the right striatum was compared (Table 4). When the total volume and neuronal density was compared between the sham-ligated groups, an effect of moderate hypothermia seemed apparent (Table 4). Yet, the total volume and neuronal density are indirect measures of neuronal survival that can be affected by tissue processing. Comparison of the absolute number of surviving neurons is preferable since this measure is shrinkage-independent. These results highlight that neuronal density and total volume data, in the absence of absolute neuronal number data, can be misleading.

\section{CONCLUSIONS}

The worked examples in this review provide evidence that indirect measures of neuronal injury or neuronal protection, in the absence of absolute neuronal number data, can lead to misinterpretations of biological processes. On this basis, it is recommended that direct measurement of the absolute number of surviving neurons is undertaken when neuroprotective therapies for brain injury are being investigated. 
Table 4. Data on the weight of the right cerebral hemisphere, the volume (V) of the right striatum, and the Nv and total number $(N)$ of medium-spiny neurons in the right striatum of Sprague-Dawley rats at postnatal day (PN) 14. On PN 7 treatment was commenced with MitoVitamin E (MitoVit-E) or its diluent. On PN 8 the rats had been subjected to neonatal cerebral hypoxia-ischemia (H/I, i.e., ligation of the right common carotid artery in the neck followed by 1.5 hrs of hypoxia). This was followed by post-treatment with either MitoVit-E or its diluent. These data are from Covey et al. (2006). For the experiment on the effect of moderate hypothermia on normal brain development, PN 7 rats had been subjected to sham-ligation of the right common carotid artery followed by no hypoxia (i.e., uninjured control rats). This was followed by immediate post-treatment for 6 hrs with either moderate hypothermia (i.e., a $5{ }^{\circ} \mathrm{C}$ decrease in whole body temperature) or normothermia, respectively. These data are from Covey and Oorschot (2007).

\begin{tabular}{|c|c|c|c|c|c|}
\hline Treatment group & $\begin{array}{l}\text { Number } \\
\text { of rats }\end{array}$ & $\begin{array}{c}\text { Weight of right } \\
\text { cerebral } \\
\text { hemisphere }(\mathrm{g})\end{array}$ & $\begin{array}{c}\mathrm{V}\left(\mathrm{mm}^{3}\right) \text { of right } \\
\text { striatum }\end{array}$ & $\begin{array}{c}\mathrm{Nv}\left(10^{4} / \mathrm{mm}^{3}\right) \text { of } \\
\text { medium-spiny } \\
\text { neurons }\end{array}$ & $\begin{array}{c}N\left(10^{3}\right) \text { of striatal } \\
\text { medium-spiny } \\
\text { neurons }\end{array}$ \\
\hline$\overline{\mathrm{H} / \mathrm{I} \text { Mito, Vit-E }(435 \mu \mathrm{M})}$ & 3 & & & & \\
\hline \multicolumn{6}{|l|}{ Pre-treatment } \\
\hline Mean & & 0.425 & 7.320 & $8.720 *$ & $667 *$ \\
\hline SD & & 0.024 & 2.410 & 3.450 & 358 \\
\hline $\mathrm{CV}$ & & 0.056 & 0.329 & 0.396 & 0.537 \\
\hline$\overline{\mathrm{H} / \mathrm{I} \text { Diluent Control }}$ & 3 & & & & \\
\hline Mean & & 0.432 & 8.490 & 14.460 & 1196 \\
\hline SD & & 0.054 & 3.110 & 2.210 & 339 \\
\hline $\mathrm{CV}$ & & 0.125 & 0.366 & 0.153 & 0.283 \\
\hline \multirow{2}{*}{\multicolumn{6}{|c|}{$\begin{array}{l}\text { Post-treatment } \\
\text { Poll }\end{array}$}} \\
\hline & & & & & \\
\hline Mean & & 0.387 & 6.680 & $10.440 \dagger$ & $699 \dagger$ \\
\hline $\mathrm{SD}$ & & 0.039 & 1.350 & 1.510 & 181 \\
\hline $\mathrm{CV}$ & & 0.101 & 0.202 & 0.145 & 0.259 \\
\hline H/I Diluent Control & 6 & & & & \\
\hline Mean & & 0.399 & 8.400 & 13.470 & 1132 \\
\hline SD & & 0.018 & 1.310 & 1.090 & 204 \\
\hline $\mathrm{CV}$ & & 0.045 & 0.156 & 0.081 & 0.180 \\
\hline Sham-ligated Control & 3 & & & & \\
\hline \multicolumn{6}{|l|}{ Moderate Hypothermia } \\
\hline Mean & & 0.453 & $10.560 \#$ & 24.790\# & 2616 \\
\hline SD & & 0.010 & 0.160 & 1.040 & 82 \\
\hline $\mathrm{CV}$ & & 0.022 & 0.015 & 0.042 & 0.031 \\
\hline Sham-ligated Control & 3 & & & & \\
\hline \multicolumn{6}{|l|}{ Normothermia } \\
\hline Mean & & 0.470 & 11.630 & 22.230 & 2585 \\
\hline SD & & 0.020 & 0.210 & 0.940 & 102 \\
\hline $\mathrm{CV}$ & & 0.042 & 0.018 & 0.042 & 0.039 \\
\hline
\end{tabular}

\section{REFERENCES}

Boyce RW, Dorph-Petersen K-A, Lyck L, Gundersen HJG (2010). Design-based stereology: introduction to basic concepts and practical approaches for estimation of cell number. Toxicol Pathol 38:1011-25.

Covey MV, Murphy MP, Hobbs CE, Smith RA, Oorschot DE (2006). Effect of the mitochondrial antioxidant, Mito Vitamin E, on hypoxic-ischemic striatal injury in neonatal rats: a dose-response and stereological study. Exp Neurol 199:513-9.

Covey MV, Oorschot DE (2007). Effect of hypothermic post-treatment on hypoxic-ischemic striatal injury, and normal striatal development, in neonatal rats: a stereological study. Pediatr Res 62: 646-51.

Galvin KA, Oorschot DE (1998). Post-injury magnesium sulfate treatment is not markedly neuroprotective for striatal medium spiny neurons after perinatal hypoxia/ischemia in the rat. Pediatr Res 44:740-5. 
Galvin KA, Oorschot DE (2003). Continuous low-dose treatment with brain-derived neurotrophic factor or neurotrophin-3 protects striatal medium spiny neurons from mild neonatal hypoxia/ischemia: a stereological study. Neurosci 118:1023-32.

Gundersen HJG (1977). Notes on the estimation of numerical density of arbitrary profiles: The edge effect. J Microsc 111:219-23.

Gundersen HJG (1986). Stereology of arbitrary particles. A review of unbiased number and size estimators and the presentation of some new ones, in memory of William R. Thompson. J Microsc 143:3-45.

Gundersen HJG, Jensen EB (1987). The efficiency of systematic sampling in stereology and its prediction. $\mathrm{J}$ Microsc 147:229-63.

Gundersen HJ, Jensen EB, Kieu K, Nielsen J (1999). The efficiency of systematic sampling in stereology-reconsidered. J Microsc 193:199-211.

Hobbs CE, Oorschot DE (2008). Neonatal rat hypoxiaischemia: long-term rescue of striatal neurons and motor skills by combined antioxidant-hypothermia treatment. Brain Pathol 18:443-54.

Oorschot DE (1994). Are you using neuronal densities, synaptic densities or neurochemical densities as your definitive data? There is a better way to go. Prog Neurobiol 44:233-47.
Oorschot DE (1996). Total number of neurons in the neostriatal, pallidal, subthalamic, and substantia nigral nuclei of the rat basal ganglia: a stereological study using the Cavalieri and optical disector methods. J Comp Neurol 366:580-99.

Oorschot DE, Galvin KA (1995). The effect of hypoxicischemic brain injury on the total number of astrocytes in the rat basal ganglia: is there hyperplasia? Proc Aust Neurosci Soc 6:234.

Perlman JM (2006). Intervention strategies for neonatal hypoxic-ischemic cerebral injury. Clin Ther 28:1353-65.

Swaab DF, Uylings HBM (1987). Density measures: parameters to avoid. Neurobiol Aging 8:574-6.

Volpe JJ (2001a). Neurology of the Newborn. $4^{\text {th }}$ ed. Philadelphia: WB Saunders.

Volpe JJ (2001b). Perinatal brain injury: from pathogenesis to neuroprotection. Mental Ret Devel Dis Res Rev 7:56-64.

West MJ (1999). Stereological methods for estimating the total number of neurons and synapses: issues of precision and bias. Trends Neurosci 22:51-61.

West MJ, Østergaard K, Andreassen OA, Finsen B (1996). Estimation of the number of somatostatin neurons in the striatum: an in situ hybridization study using the optical fractionator method. J Comp Neurol 370:1122. 